\title{
Anatomie der Pleura
}

\author{
Hendrik Dienemann
}

\section{Inhalt}

Die linke und rechte Pleurahöhle (Cavitas thoracis) stellen, vergleichbar mit der Peritoneal-oder Perikardhöhle, in sich geschlossene seröse Säcke dar. Der Pleuraraum ist jedoch ein kapillärer Spalt, da die Lunge von medial her den Pleurasack einstülpt. Somit entsteht ein viszerales Blatt (Pleura visceralis), das die Lunge bedeckt. An der Umschlagfalte in Umgebung des Lungenhilus geht es in das äußere Blatt (Pleura parietalis) über, das mit der Innenfläche der Thoraxwand verbunden ist. Die Anheftung der Pleura parietalis erfolgt durch eine Schicht von subserösem Bindegewebe, die sog. Fascia endothoracica. Diese Schicht, die den Brustraum beiderseits des Mediastinums auskleidet, ist vorne sehr dünn, wird nach dorsal hin dicker und hat lediglich im Bereich der Pleurakuppel, wo sie sich nicht scharf von der Umgebung trennen lässt, die Eigenschaften einer Faszie. Am Sternum und an der Wirbelsäule geht die Fascia endothoracica in das lockere Bindegewebe des Mediastinums über. Die praktische Bedeutung dieser sog. Faszie liegt für den Chirurgen darin, dass die Pleura parietalis in der Schicht der Fascia endothoracica abgelöst werden kann.
Die Pleura visceralis ist mit der Lungenoberfläche fest verwachsen. Sie setzt sich in die Interlobärspalten der Lungen fort. Die Pleura parietalis geht am Lungenhilus und im Lig. pulmonale aus der Pleura visceralis hervor und überzieht als Pleura costalis Rippen, Wirbelkörper und die Rückfläche des Brustbeins, als Pleura diaphragmatica die obere Zwerchfellfläche und als Pleura mediastinalis das Mediastinum. Kranial überragt die Cupula pleurae die 1. Rippe und nimmt die Lungenspitze auf. Bei maximaler Exspiration gehen die Pleuraspalten weit über die Lungenränder hinaus. So entstehen der Recessus costomediastinalis am Übergang der Pleura costalis in die Pleura mediastinalis, der Recessus phrenicomediastinalis am Übergang der Pleura diaphragmatica in die Pleura mediastinalis und der praktisch wichtigste, der Recessus costodiaphragmaticus, am Übergang der Pleura costalis in die Pleura diaphragmatica. Dieser ist in der Axillarlinie bis $8 \mathrm{~cm}$ hoch und wird auch bei maximaler Inspiration niemals vollständig entfaltet.

H. Dienemann ( $\square)$

Chirurgie Thoraxklinik Heidelberg, Universitätsklinikum Heidelberg, Gräfelfing, Deutschland 